\title{
Theoretical and experimental researches on development of new construction of wind-driven generator with flux concentrator
}

\author{
Koshumbayev Marat ${ }^{1}$, Yerzhan Assem ${ }^{2,}{ }^{*}$, Myrzakulov Bakhytzhan ${ }^{3}$, Kvasov Peter ${ }^{4}$ \\ 1, 2, 3, 4 Kazakh Research Institute of Power Engineering Named After Academician SH. CH. Chokin \\ Joint-Stock Company, Almaty 050012, Republic of Kazakhstan
}

\section{Index Terms}

Wind-Driven Generator Vertical Axis of Rotation

Turbulence Turbulent Flows

Wind Turbine

Blades

Received: 21 May 2016

Accepted: 1 June 2016

Published: 21 June 2016

\begin{abstract}
The subject of research is the new construction of a wind-driven generator with a flux concentrator. Tasks to research air flow motion in swirling wind turbines, to develop the methodology of wind turbine calculation and determination of its capacity with initial and boundary conditions are stated in the article. In the work literature, patent review and analysis of mathematical methods of existing hydro turbine constructions were made. As a result of the research calculation methods of wind turbine and determination of its capacity with initial and boundary conditions were developed. Also, an experimental stand was made. Main constructive and technical, and operational characteristics: concentrator's convergent channels are curvilinear in view and are described by logarithmic dependence. The construction's novelty consists of a flow concentrator with curvilinear convergent channels tangentially connected with a ventilation pipe.
\end{abstract}

(C) 2016 The Author(s). Published by TAF Publishing.

\section{INTRODUCTION}

Nowadays nobody has doubts that the usage of sun, wind and other kinds of power will constantly grow. Wind engines theory, which is developing for several decades, is far from its end. Indeed, we can say that there are no exact and reliable enough methods of aerodynamic calculation of wind-power units.

An important advantage of wind-power units is the fact that they come under the cleanest power sources from the ecological point of view.

\footnotetext{
* Corresponding author: Yerzhan Assem

E-mail: yerzhanassem@gmail.com
}

Modern wind engines are rather simple in maintenance. They can be mounted in any remote regions, where it is difficult to deliver fuel and other power sources. Further development of wind power is impossible without wide development of scientific researches in this direction.

Up to XIX century researches of D. Bernoulli, L. Euler, d'Alembert and other famous scientists did not go beyond general consideration of air flow in environments of wind wheel. First attempts of analytical description of the flow, which are in their character near to that realized during flow through blades of wind wheel, were connected with researches of propeller blade [1].

In the last quarter of $\mathrm{XX}$ century questions of wind 
engines' aerodynamics were generalized and stated in a number of publications [2, 3]. Obsolescent infrastructure of power generation in Kazakhstan and urgent necessity in its replacement for support of acceptable quality levels and reliability of electric power supply are the possibilities to use wind power [9]. Nowadays Kazakhstan is one of the largest carbon emission sources in the world per capita. There is strong dependence (approximately 85\%) from generation of electric power with coal usage. Pass to moderate coal usage during generation of electric power is a stimulus for development of wind power. Very low carbon intensity from wind stations attracts investors, because developing mechanisms on climate changing allow making such projects commercially implementable [10].

\section{MAIN PART}

There is a wind electric station construction consisting of air flow concentrator in the form of tent with ventilation pipe and cone with concave surface, wind wheel, generator and battery (Fig. 1).

Cone and tent are connected to each other by vertical divisions, which form convergent air channels. Wide side is for air flow input, narrow side is for supply of accelerated flow in vertical ventilation pipe, where wind wheels are mounted on vertical shafts. To disadvantages of wind electric station we can attribute low efficiency; flow concentration does not happen in the operating surface of wind wheel blade and it leads to considerable decrease of engine efficiency and time resources of mechanism joints' operation.
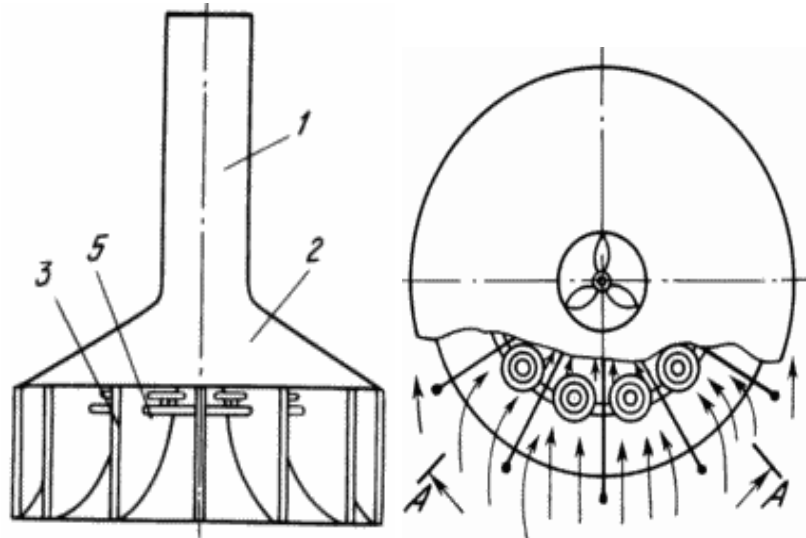

Fig. 1. Construction of wind electric station (patent of Russian Federation RU2062353)

Another construction of wind electric station (Fig. 2) has thermal component, in other words flow is preliminary heated and supplied under the tent. Temperature gradient makes air motion, which drives generator's blades.
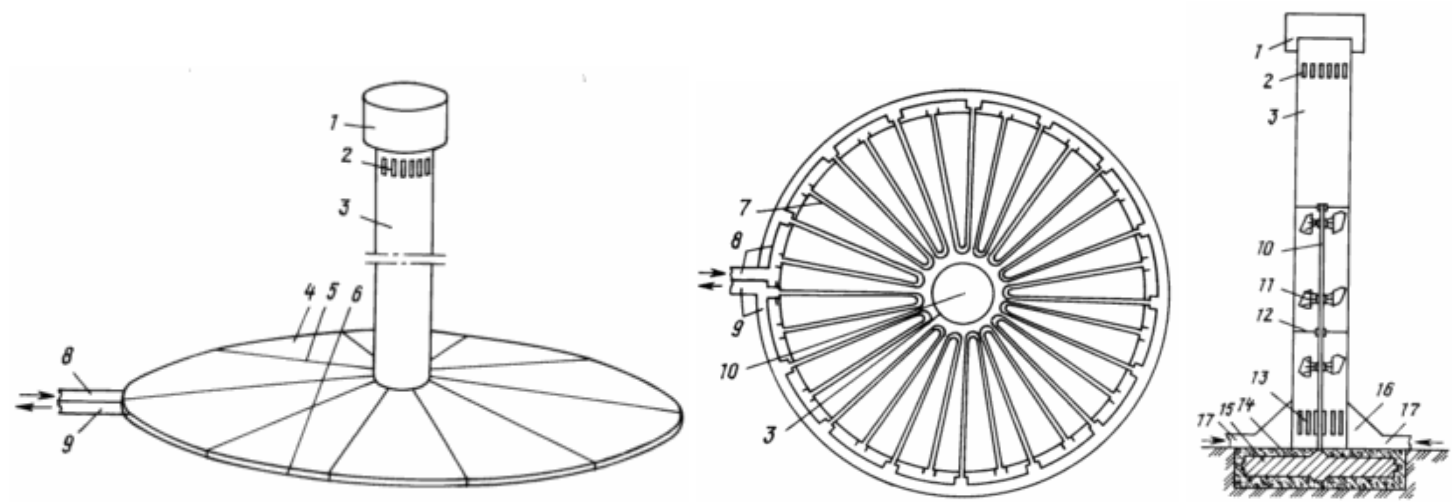

Fig. 2. Thermal wind electric station (patent of Russian Federation RU2070660)

On vertical shaft of generator wind wheels with blades are fixed inside the pipe. For air flows swirling there are vertical cuts with guiding shoulder blades in the low part of the pipe.

Disadvantages of both famous analogues are: low efficiency because of ineffective flow concentration and its influence on blade's back drive, low reliability, the absence of air heating system, necessity in additional flaps for air blow turn.

Wind generators with vertical spinning axis have important advantages before wind generators with horizontal axis location. For them there is no necessity in devices for wind orientation, the construction is simplified and gyroscopic loads causing additional loads in blades, transmission system and other installation elements with horizontal spinning axis are decreased 
Essential disadvantages of such installations are:

a) larger susceptibility of them to fatigue breakdowns because of more frequent autowave processes appearing in them;

b) torque pulsation leading to undesirable pulsations of generator outputs.

c) problems of flexible shaft.

Because of that a lot of wind electric generators are made according to horizontal-axis scheme. However researches of different types of horizontal-axis installations continue.

The capacity of wind-driven generator depends on effectiveness of air flow usage. One of the methods of its increasing is the usage of special concentrators (intensifiers) of air flow. For horizontal-axis wind-driven generators different variants of such concentrators are developed or offered [11]. It can be diffusers or confusors (deflectors), which guide air flow from the area, to larger rotor's sweeping area, on wind wheel and some other devices. Concentrators do not have wide distribution in industrial generators.

The case is that on practice the confusor's efficiency is less than expected. It is almost impossible to increase wind speed more than on $20-30 \%$, because with large air flow speed rates air swirls are formed in confusor and they prevent air passing.

There is also swirling wind generator, the working principle of which is based on swirling processes. Special diffuser construction allows strong air flow, which transforms into upstreaming swirl inside the parabolic column. Such aggregates are more efficient but also not very widespread.

Swirling wind generator has capacity from 2 to $100 \mathrm{~kW}$ and more. Wind orientation is needed for it. There are no slow-speed rotating blades (scapular turbine is used), there are no radio interferences and infra-sound on frequencies dangerous for biological objects (about $7 \mathrm{~Hz}$ ). It has smaller dimensions from several turret-type sections, which considerably economize means on mounting and decreases generator dimensions in whole. Swirling wind generators have considerable advantages in comparison with blade wind generators.

Devices for wind energy transformation into electrical energy without application of motion parts are developed. To them is related, for example, the device, in which the process of matters cooling in wind flow is used for electric energy generation on the base of thermoelectric Thomson effect.

In case of swirling wind turbine air is heated as a result of compression in confusors and by this creates additional difference for speed intensification of gases coming from the top part of the pipe.

In offered solution inside of concentrator between tent and cone curved guiding walls forming curved air channels are mounted, curved blades are fixed on the generator's axis. Curvilinearity of guiding walls' surfaces and wind wheel blades is given by logarithmic dependence.

Wind aggregate, which includes concentrator consisting of tent and cone, wind wheel with blades, generator with vertical shaft, ventilation pipe are offered. By this curved
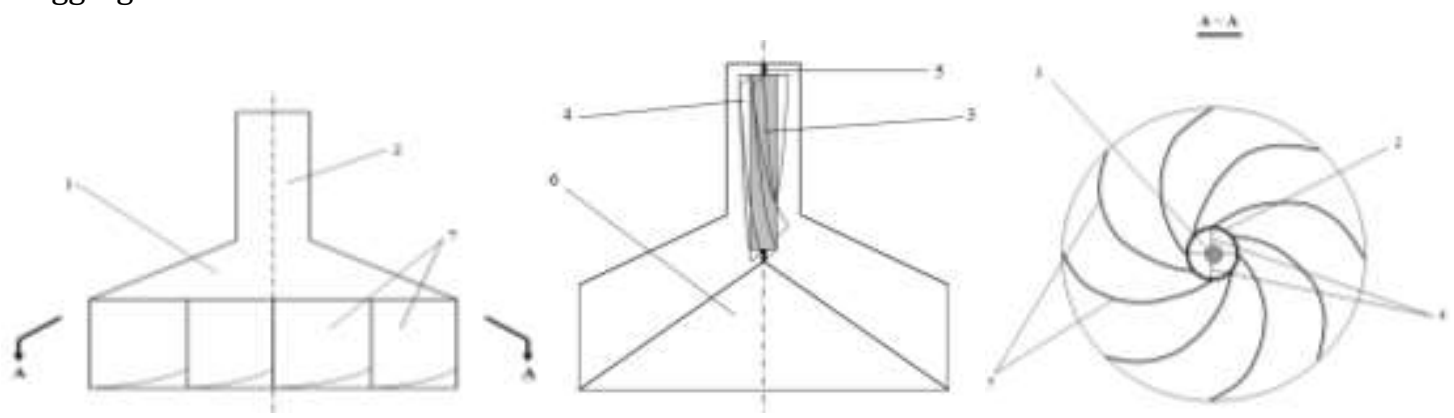

Fig . 3. Swirling wind station

guiding walls connecting tent with cone and forming curved convergent air channels, which are tangentially directed to ventilation pipe, are placed in concentrator. Wind wheel is made in the form of generator's enclosure, on the outer side of which curved blades are mounted. In addition, generator's shaft is fixed by one end in the cone centre and by another end-in the top of the ventilation pipe. Outer sides of the tent and ventilation pipe are painted in black color.

Air flow, coming into concentrator, is heated and moves on curved channel to ventilation pipe. Channel's convergence allows increasing the flow speed and tangentially guides it to ventilation pipe. The outlet of each channel is directed only on one curved blade, in other 
words flow action is directed to exclude its impact on back run of blades.

Technical result is achieved by the fact that offered device, as well as famous device, activates air flow concentrator in the form of the tent with ventilation pipe and cone with concave surface, wind wheel, generator.

However, in contrast with famous device, curved guiding walls forming curved air channels are mounted inside of the concentrator between tent and cone, Generator is placed inside in the centre of wind wheel, curved blades are fixed on generator's surface. Curvilinearity of guiding walls and wind wheel blades is given by logarithmic dependence.

In the centre of the tent the ventilation pipe is mounted. Inside the pipe the wind wheel in the form of generator is placed. On the outer side of this wheel curved blades are fixed. Generator's shaft is fixed in the cone centre and in the top part of the pipe. Curved guiding walls, which form curved convergent air channels providing tangential air flow supply in ventilation pipe, are situated in flow's concentrator between tent and cone. Outside surfaces of tent and ventilation pipe are painted in black color. Curving of guiding walls and blades is given by logarithmic dependence.

The device works in the following way. Air flow comes to concentrator and moves between tent and cone along curved guiding walls. Under the influence of solar power tent and pipe are heated, increasing the temperature of air flow. Heated air moves on curved convergent channel and its speed increases by means of temperature rising and decreasing of clear opening. Channel's outlet is directed in such a way that air flow tangentially comes into ventilation pipe and swirling motion forms in it. In addition swirling flow makes hydrodynamic pressure only on one blade, so air motion does not influence on its back run at all. It means that blades do not have resistance. Generator's placement in ventilation pipe also decreases flow's clear opening and increases its speed. Swirling motion energy is passed on curved blades. Owing to the direction of spinning axis along the flow blades do not experience frontal impact by air flow and console tension even in case of unequal approach of air flow to concentrator channels' inlets and by changing of its direction. Blades, when rolling, pass torque to generator, which transforms mechanical rolling energy into electrical one. Air flow, coming into concentrator, is heated and moves spirally to the centre. Spiral air motion is determined by guiding walls

reduce the energy of possible tornado. Together with it, the offered solution will allow transforming the swirl and blades configuration. The direction of angular speed of the swirl depends on walls curving. With concentrator's radius increasing the aggregate's capacity increases.

The offered construction can be mounted not only on land but also on the surface of sea or ocean. Efficiency of this construction is evident especially for remote places, where electrical energy by usual method is impossible. Offered solution allows increasing of aggregate's capacity by means of impact elimination of resistance forces and blades mounting on the outer side of generator. By this, reliability of aggregate's operation and its operational characteristics are increased.

To additional advantages of this construction can be referred: blades vibration absence, elimination of impact force on blades by air flow.

Conclusion. The usage of offered wind aggregate provides the achievement of technical result: air, coming into concentrator, is heated and with the help of curved guiding walls gets spinning motion. Temperature increasing and decreasing of flow's clear opening also contributes to its stable accelerated spinning motion. Swirling air motion leads to the spinning of blades and generator itself. In addition blades on back run do not suffer from resistance forces. Curved blades provide gradual flow slip, accept its impact and gives spinning to generator. There is no console load on blades and the increasing of its length does not arouse the growth of flow's impact force as in case of prototype, and consequently there is no tension moment in places of blades fixation with generator. As there is no console load, the thickness of blades can be very small, it decreases construction's materials intensity. Application of the offered construction improves work conditions of blades, decreases fatigue stress and increases reliability of its work. Blades are fixed on the outer side of the generator and spinning energy is passed to him without additional mechanisms. All these facts simplify construction, provide quick mounting or dismantling of aggregate, visual examination and elimination of defects during its operation.

Conclusions. Offered solution is one of the variants of artificial ventilation of air basin of large metropolises. If stating the question wider, it is possible to create constructions, which will reduce tornados' energy, which is the real catastrophe for countries of the Caribbean and other countries (for example, Japan, China). Offered constructions will create some swirling flows, which will energy into electric current, which means that new construction will allow not only creation of swirling 
motion but also use its energy.

\section{REFERENCE}

[1] G. N. Abramovich, Theory of Turbulent Streams. Mosco, Russia: Fizmatgiz, 1960, p. 716.

[2] L. Yaras, L. Goffman, A. Yaras and G. Obermayer, Wind Power (Translation from English; Under the editorship of Y. I. Shefter \& M. Mir), 1982.

[3] R. E. Wilson, "Wind-turbine aerodynamics," Journal of Industrial Aerodynamics, vol. 5, no. 4, pp. 357-372, 1980.

[4] R. O. Preuss, E. O. Sussiu and L. Morino, "Potential aerodynamic analysis of horizontal-Axis windmills," pp. 1132-1140, 1977.

[5] R. E. Wilson, P. B. S. Lissaman and S. N. Walker, "Aerodynamic performance of wind turbines," Oregon State University, Corvallis, OR, 1976.

[6] A. N. Kolmogorov, "Equations of turbulent motion of incompressible fluid," Izvestiya Akademii Nauk: Seriya
Khimicheskaya, vol. 6, pp. 56-58, 1991.

[7] J. O. Hinze, Turbulence: An Introduction to its Mechanism and Theory. New York, NY: McGraw-Hill, 1963.

[8] J. H.Ferziger, "Large eddy simulations of turbulent flows," AIAA Journal, vol. 15, no. 9, pp. 1261-1267, 1977.

[9] "Modern Condition and Perspectives of Development of Wind Power," Magnitogorsk, Russia: Informenergo, 2000.

[10] E. M. Perminov, Wind Power Development in European Countries. n.d.

[11] E. M. Fateev, Wind Power Installation: Present Condition and Possible Lines of Development. Moscow, Russia: Gosenergoizdat, 1946, p. 520.

[12] [Online]. Available: http://goo.gl/THL4h1

— This article does not have any appendix.- 